Rhode Island College

Digital Commons @ RIC

$5-13-2017$

\title{
Comparing the Canadian Cervical Spine Rule and Radiology Confirmed Cervical Spine Injury in the Emergency Department
}

Ashley Claire Arnold

Rhode Island College

Follow this and additional works at: https://digitalcommons.ric.edu/etd

Part of the Nursing Commons

\section{Recommended Citation}

Arnold, Ashley Claire, "Comparing the Canadian Cervical Spine Rule and Radiology Confirmed Cervical Spine Injury in the Emergency Department" (2017). Master's Theses, Dissertations, Graduate Research and Major Papers Overview. 191.

https://digitalcommons.ric.edu/etd/191

This Major Paper is brought to you for free and open access by the Master's Theses, Dissertations, Graduate Research and Major Papers at Digital Commons @ RIC. It has been accepted for inclusion in Master's Theses, Dissertations, Graduate Research and Major Papers Overview by an authorized administrator of Digital Commons @ RIC. For more information, please contact digitalcommons@ric.edu. 
COMPARING THE CANADIAN CERVICAL SPINE RULE

AND RADIOLOGY CONFIRMED CERVICAL SPINE INJURY IN THE

EMERGENCY DEPARTMENT

\author{
by \\ Ashley Claire Arnold, BSN, RN, CEN \\ A Major Paper Submitted in Partial Fulfillment \\ of the Requirements for the Degree of \\ Master of Science in Nursing \\ in
}

The School of Nursing

Rhode Island College

2017 


\begin{abstract}
In the United States and Canada, more than 13 million trauma patients are at risk for cervical spine injury (CSI). To date, providers use computerized tomography (CT) to rule out CSI in these patients. Cervical spine immobilization with the use of rigid cervical collars can be uncomfortable, delays hospital transport, and has a modest morbidity. The Canadian Cervical Spine Rule (CCR) is a screening tool used in emergency departments (ED) as a screening tool to rule out CSI to avoid unnecessary CTs. The purpose of this project is to complete a retrospective chart review to evaluate if emergency departments can apply the CCR to reduce cervical spine radiographs in the adult patient with suspected CSI. The study was a retrospective chart review, which took place at a level 2 trauma center emergency department in a community teaching hospital reviewing patient records and radiological findings in relation to the potential benefit of using the CCR in CSI assessment. The sample was a non-probability convenience sample of patients who have had a cervical spine radiograph in the ED. One hundred charts were reviewed and 60 patient records were included in the study. Of the 60 records included in the study, half met high risk criteria requiring cervical spine radiography. Of the 30 records meeting low risk criteria, only 2 charts had complete documentation including neck range of motion (ROM). One had normal ROM and one was unable to actively rotate neck. There were 8 records that documented "normal range of motion" but did not specifically note normal neck ROM. It is difficult to definitively state that $28 \mathrm{CT}$ s could have been prevented, as there was inadequate neck range of motion documentation. There were no cervical spine injuries detected by CT in this study. As with previous studies, the data shows that the CCR maintains its sensitivity but has low specificity. It remains that clinicians still rely on radiology evidence to rule out CSI rather than assessment tools.
\end{abstract}




\section{Table of Contents}

Background/Statement of the Problem ............................................................... 1

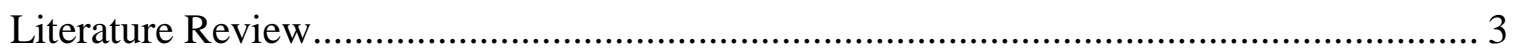

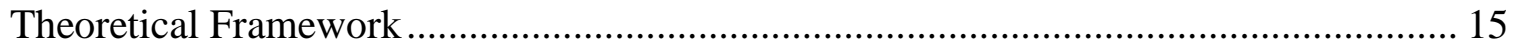

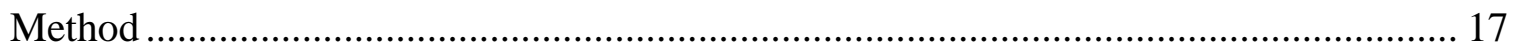

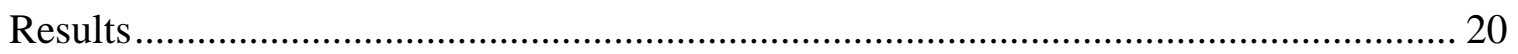

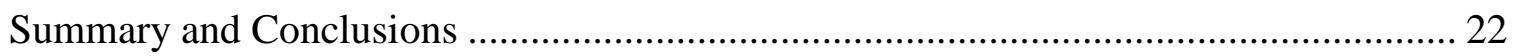

Recommendations and Implications for Advanced Nursing Practice ........................... 24

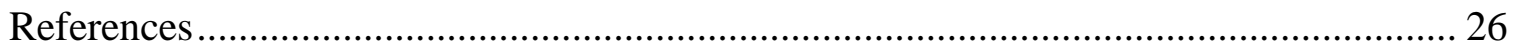

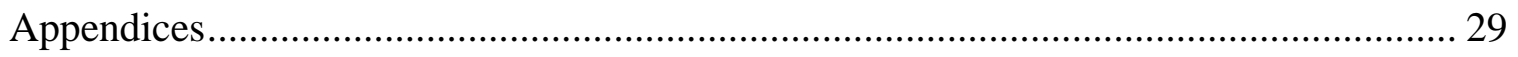




\section{Background/Statement of the Problem}

Cervical spine injuries have significant morbidity and mortality ranging from chronic pain to paralysis and even death. Due to the possible detrimental effects of a spinal cord injury, the standard of care by emergency medical services is to immobilize the cervical spine of all trauma patients until injury can be ruled out with clinical assessment or radiography. The American Association of Neurologic Surgeons currently does not recommend radiographic assessment of the cervical spine in patients who are awake, alert, not intoxicated, and do not have significant neck pain or distracting injuries. Radiographic study of the cervical spine of every trauma patient is costly and results in significant radiation exposure to a large number of patients, very few of whom will have a spinal column injury (Hadley \& Walters, p.91, 2001).

In the United States and Canada, more than 13 million trauma patients are at risk for cervical spine injury. To date, physicians use computerized tomography (CT) to rule out cervical spine injury. Of these 13 million, only $2 \%$ of radiographs are found to have a cervical spine fracture. This leaves $98 \%$ of these patients with the unnecessary radiation and cost of a CT scan (Stiell et al., 2009).

The cost of health care in the US is rapidly rising. In 2014, US health care spending reached $\$ 3.0$ trillion, accounting for $17.5 \%$ of the gross domestic product (California Health Care Foundation, 2016). The US spends more money on healthcare than any other country in the world. Yet the US has inferior health outcomes, shorter life expectancy, and greater prevalence of chronic conditions than other highly developed countries (Squires \& Anderson, 2015). 
Emergency department overcrowding has increased dramatically over the past 20 years. Providers are ordering more diagnostic testing for fear of litigation, uninsured patients are seeking primary care from emergency departments, and hospitals around the country are closing. According to the Institute of Medicine, ED visits have increased 26\% while the number of EDs decreased by 425 between 1993 and 2003. Overcrowding has also led to a significant increase in ambulance diversion, which leads to delays in care and treatment for ill patients, placing the patient at significant risk (IOM, 2006, p. 31). Increased unnecessary procedures and testing can lead to prolonged ED length of stay and increased cost to the patient.

There are currently two clinical decision rules, the Canadian C-Spine Rule (CCR) and the National Emergency X-Radiography Utilization Study (NEXUS) available to assess the need for imaging in patients with suspected cervical spine injury. Numerous studies have shown that the use of the CCR and NEXUS can safely decrease the number of cervical spine radiographs used to rule out an acute cervical spine fracture. In one study, imaging rates decreased $42 \%$ without missing an injury with use of the CCR (Michaeff, Maher, Verhagen, Rebbeck, \& Lin, 2012). However, the use of the CCR is not consistently utilized in Emergency Departments around the United States. This quality project aims to review medical records from the Emergency Department to determine how many cervical spine radiographs could have safely been avoided if the CCR was utilized. 


\section{Literature Review}

A literature review was performed using CINHAL and OVID. The search included the key words cervical spine, clearance, Canadian Cervical Spine Rule, NEXUS, Emergency Department, computerized tomography, wait times, cost, crowding, and length of stay. The search included articles from 2000 through 2016.

\section{Spinal Cord Injury}

The human spine is made up of three sections, the cervical, thoracic, and lumbar spine. The individual bones are called vertebra. The top seven vertebra make up the cervical spine. The spinal cord rests within the spinal column. Disruptions in the spinal column can ultimately injure the neuromusculature throughout the body leading to paralysis and dysfunction. A diagram of the cervical spine is depicted below in Figure 1 (Spinal Cord Injury Model System, 2015).

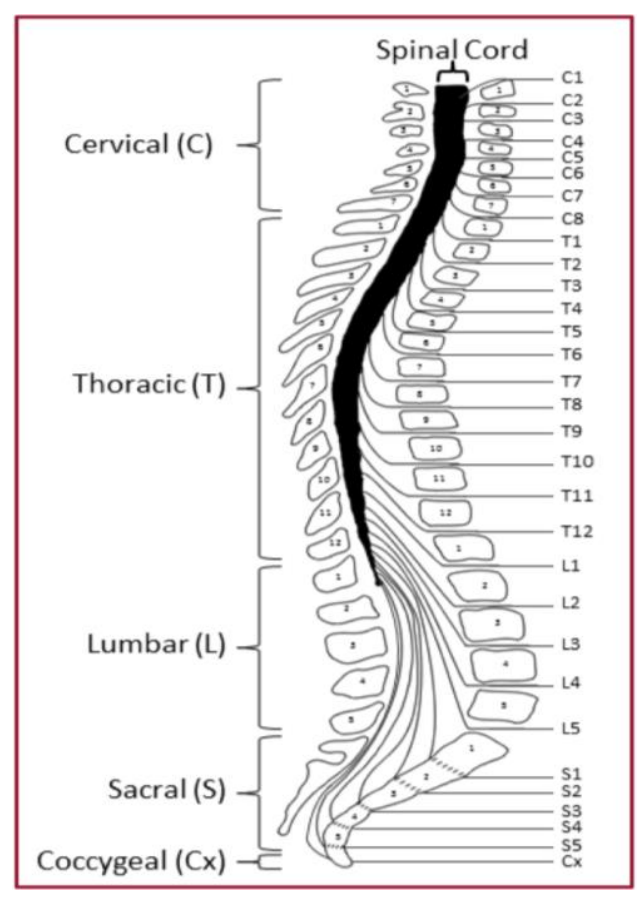

Figure 1. The Spinal Column 
Cervical spine injuries resulting in spinal cord injuries (SCI) are rare yet detrimental, resulting in long-lasting and devastating physical disabilities. Cervical spine injury can be in the form of a fracture or dislocation. According to the Spinal Cord Injury Model System (2015), motor vehicle accidents account for approximately half of all spinal cord injuries. Speeding, alcohol intoxication, and lack of proper seatbelt use increases the risk of injury in a motor vehicle accident. Other causes of spinal cord injuries include falls, violence, such as gunshot wounds, and sporting activities (Spinal Cord Injury Model System, 2015).

Spinal cord injuries frequently lead to permanent disability of those affected. Currently, there is no "cure" for spinal cord injury. The extent of their disability and paralysis is directly related to the level of the SCI. Patients with cervical spinal cord injuries have the greatest disability compared to those with injuries of the thoracic or lumbar spine. For example, patients with injury at the C1-C3 level only have limited movement of the head and neck. Patients with injury at the C3-C4 level have control of their head and neck and may be able to shrug their shoulders. At most, patients with C7 injuries can move their head, neck, shoulders, arms and wrists. Patients with SCI spend the rest of their lives in physical therapy and rehabilitation with very little chance of functional improvement (Spinal Cord Injury Model System, 2015).

\section{Emergency Department Overcrowding}

According to the Institute of Medicine (2006), ED visits have increased 26\% while the number of EDs decreased by 425 between 1993 and 2003. Up to 91\% of EDs reported overcrowding as a problem in a national survey. Overcrowding leads to 
increased stress on patients and providers alike, and can lead to impaired quality of care and potential errors. The significant ED overcrowding has led to many departments boarding admitted patients for up to 48 hours or more. Overcrowding has also led to a significant increase in ambulance diversion (IOM, 2006, p.31). Ambulance diversion is the practice of rerouting ambulances away from the closest emergency department often delaying timely care delivery (Cuong Pham, Patel, Millin, Kirsch, and Chanmugam, 2006, p. 1220). Over 500,000 ambulances were diverted in 2003, which leads to delays in care and treatment for ill patients, placing the patient at significant risk (IOM, 2006, p. 31).

\section{Cost of Emergency Department Healthcare}

A study published in the American Medical Association in 2014 notes that the mean cost of an Emergency Department visit has increased 240\% from $\$ 560$ to $\$ 1354$ from 2003 to 2011 (p. 2). The researchers related the increase of healthcare spending to the overuse of diagnostic tests, treatments, and hospitalizations that emergency clinicians order (p. 2). The purpose of their study was to create a list of five tests, treatments, and disposition decisions that are of little value, amendable to standardizations, and are actionable by emergency medicine physicians (p. 1). The number one recommendation was "do not order computed tomography (CT) of the cervical spine for patients after trauma who do not meet the National Emergency X-ray Utilization Study (NEXUS) low criteria or the Canadian C-Spine Rule" (Schuur, Carney, Lyn, Ali, Michael, Ross, \& Venkatesh, 2014, p. 3). 


\section{Current C-Spine Assessment Guidelines}

The American Association of Neurologic Surgeons (AANS) developed a consensus statement in 2001 regarding the protocol for cervical spine immobilization (Hadley \& Walters, 2001). The AANS suggests that all patients with a mechanism of injury having the potential to cause cervical spine injury be immobilized at the scene and during transport using a rigid cervical collar and supportive blocks on a backboard with straps (p. 18). It is estimated that $3 \%$ to $25 \%$ of spinal cord injuries occur after the initial traumatic insult, either during transport or during early management (p. 18). The AANS does not recommend radiographic assessment of the cervical spine in patients who are awake, alert, not intoxicated, and do not have significant neck pain or distracting injuries. Radiographic study of the cervical spine of every trauma patient is costly and results in significant radiation exposure to a large number of patients, very few of whom will have a spinal column injury (Hadley \& Walters, 2001, p. 91).

According to Hadley and Walters (2001), asymptomatic patients following trauma are defined as those patients who meet all of the following criteria:

1. Neurologically Intact Glascow Coma Score of 15, without the following: a) disorientation to person, place, or time; b) inability to remember 3 objects at 5 minutes; c) delayed or inappropriate response to external stimuli; or d) any focal motor or sensory deficit.

2. NOT Intoxicated. Intoxication as defined as: a) a recent history of intoxication or intoxicating ingestion; b) evidence of intoxication on clinical examination; or c) laboratory evidence for the presence of drugs which alter the level of alertness, including alcohol greater than $0.08 \mathrm{mg} / \mathrm{dl}$. 
3. Absent neck pain or midline tenderness. Midline tenderness is present if the patient complains of pain on palpation of the posterior midline neck from the nuchal ridge to the first thoracic vertebra.

4. No associated injury that is distracting to the patient. Significant distracting injuries have been defined as: a) long bone fractures; b) visceral injuries requiring surgical consultation; c) large lacerations, degloving, or crush injuries; d) large burns; and e) any other injury which might impair the patient's ability to participate in a general physical, mental, and neurological examination.

Based upon these criteria, approximately one third of trauma patients evaluated in emergency rooms or trauma centers are asymptomatic (Hadley \& Walters, 2001, p. 93-94).

\section{Massachusetts Guidelines}

The Massachusetts Department of Public Health created spinal assessment guidelines in 2015 for first responders. According to these guidelines concerning mechanisms that may result in spinal column injury include: fall from over 3 feet, including adult fall from standing, or greater than 5 stair steps; motor vehicle collision (MVC) at greater than 30mph, rollover or ejection; motorcycle, bicycle, or other mobile conveyance, or pedestrian-vehicle accident; diving or axial load; or electrical shock (p.88). Symptoms of spinal column injury may include: posterior neck or back pain or tenderness; paresthesias or loss of sensation in extremities; weakness or paralysis of extremities (p.88). Conditions placing patients at risk to not perceive or complain of the symptoms of spinal column injuries: Altered mental status due to disease, injury, intoxication, or other causes; inability to adequately communicate; history of cervical 
spine injury or abnormality or conditions causing fragile bones; distracting injury (such as long-bone fracture); age extremes (including $>65$ years of age) (p.88). The Massachusetts guidelines state "individuals sustaining lesser injuries, patients who do not have symptoms of spinal column injury and do not experience a condition that would impair the patient's ability to perceive or communicate symptoms of spinal column injuries do not require spinal immobilization" (p. 88) (Massachusetts Department of Public Health, 2015).

\section{Decision Making Tools}

Currently there are two clinical decision rules, the Canadian C-Spine Rule (CCR) (Appendix A) and the National Emergency X-Radiography Utilization Study (NEXUS) (Appendix B) available to assess the need for imaging in patients with cervical spine injury (Michaleff, Maher, Verhagen, Rebbeck, \& Lin, 2012).

The CCR can be utilized for alert Glascow Coma Scale (GCS) of 15 and stable trauma patients where cervical spine injury is a concern. The CCR cannot be applied to non-trauma cases, GCS $<15$, unstable vital signs, age less than 16 years, acute paralysis, known vertebral disease, or previous surgery of cervical spine. To utilize the CCR, the provider first evaluates for any high risk factors that mandate radiography. These high risk factors include age greater than or equal to 65 years of age, paresthesias in the extremities, or dangerous mechanism of injury. Dangerous mechanism of injury includes fall from elevation greater than or equal to three feet or five stairs, axial load to head MVC at high speed greater than $100 \mathrm{~km} / \mathrm{hr}$, rollover, ejection, motorized recreational vehicles, bicycle struck or collision. If the patient meets any high risk factor, the patient 
will require imaging to rule out cervical spine injury. If the patient does not have any high risk criteria, the provider then assesses for any low risk factor that allows for safe assessment of range of motion. The low risk factors include simple rear end motor vehicle collision, sitting position in emergency department, walking at any time, delayed onset of neck pain, or absence of midline cervical spine tenderness. The patient only requires one of these low risk factors to allow safe assessment of range of motion. If the patient has zero low risk factors, imaging of the cervical spine will be required. If the patient has any low risk factor, the provider will then ask the patient to actively rotate their neck 45 degrees to the left and right. If the patient is able to do so, the patient does not require radiography. If the patient is unable to actively rotate their neck, they will require imaging of the cervical spine (Stiell et al., 2009).

The NEXUS low-risk criteria is another tool providers can use to determine if patients in the emergency department require cervical spine imaging. According NEXUS criteria, cervical spine radiography is indicated for patients with neck trauma unless they meet all of the following criteria, no posterior midline cervical-spine tenderness, no evidence of intoxication, a normal level of alertness (GCS=15), no focal neurologic deficit, and no painful distracting injuries (Michaleff et al., 2012).

\section{Evaluating the CCR and NEXUS criteria}

The accuracy of the Canadian C-Spine rule was compared to the NEXUS criteria in a systematic review by Michaleff et al., (2012). The purpose of this systematic review was to describe the quality of research evaluating the CCR and NEXUS. In this systematic review, 578 studies were viewed and a group of 15 were compiled using an 
electronic search of CINAHL, Embase and MEDLINE. Eight of the studies evaluated the CCR alone and 6 studies evaluated NEXUS alone. Only one of the studies was a direct comparison. Only cohorts of patients with blunt trauma and for whom clinically important cervical spine injury detectable by diagnostic imaging were evaluated by the CCR or NEXUS or both. It was found that the CCR had a sensitivity of 0.9 to 1 and a specificity of 0.01 to 0.77 with a low false negative rate of $0 \%$ to $0.11 \%$. On average, imaging rates decreased $42 \%$ without missing an injury with use of the CCR. The NEXUS criteria was found to have a sensitivity of 0.83 to 1 and specificity from 0.02 to 0.46 with a higher false negative rate of $0 \%$ to $1 \%$. On average, imaging rates decreased $30.9 \%$ without missing a clinically important cervical spine injury. For both rules, a negative test was more informative. A low specificity with a high level of false positives was found, meaning many patients were still undergoing CT. In the comparison study, the CCR had better diagnostic accuracy for sensitivity and specificity and would have reduced imaging rates by $44 \%$ while NEXUS would have reduced rates by $36 \%$. One limitation to this study was that heterogeneity prevented pooling of the results so the findings of the review are based on individual studies and only one direct comparison (Michaleff, et. al., 2012).

A retrospective cross-sectional study was published in 2016 in the Journal of Trauma and Acute Care Surgery. The purpose of this study was to review the prevalence of C-spine fractures, as well as review the appropriateness of imaging by NEXUS and CCR criteria, and estimate radiation dose exposure and costs associated with C-spine imaging studies. In this study 760 patients met the inclusion criteria. Inclusion criteria was a documented evaluation in the ED for a positive history of ground level fall (GLF). 
A GLF is a fall of less than 3 feet or 5 stairs. The patient must have also had a C-spine CT. Exclusion criteria included a fall greater than 3 feet or 5 stairs, a fall accompanied by axial load (diving, MVC at high speed greater than $100 \mathrm{~km} / \mathrm{hr}$ ), age less than 16 years, a patient found down at the scene or unknown etiology/indeterminate mechanism, and other mechanisms of trauma such as penetrating gunshot/stab wound, MVC, pedestrian struck by a moving vehicle, or a bicycle accident (p. 340). Seven C-spine fractures were identified. All fractures were identified by the NEXUS and CCR with $100 \%$ sensitivity. Of all the imaging studies performed, $69 \%$ met NEXUS indications for imaging and 50\% met CCR indications. C-spine CT scans in patients not meeting the NEXUS or CCR indications for radiography were associated with costs of $\$ 15,500$ to $\$ 22,000$ by NEXUS and $\$ 14,600$ to $\$ 25,600$ by CCR during this 6-month period. One limitation of this study was that it only included patients with a ground level fall (Benayoun, Allen, Lovasik, Uriell, Spandorfer, and Holder, 2016).

\section{The Canadian Cervical Spine Rule}

In 2009, Stiell and his colleagues completed a study with the purpose of evaluating the effectiveness of an active strategy to implement the validated CCR into multiple emergency departments. For this quantitative study, they completed a matched pair cluster randomized trial in 12 Canadian emergency departments. Their study population included 11,824 alert and stable adults above the age of 16 with blunt trauma to the head or neck. The study found a relative reduction in imaging of $12.8 \%$ in the intervention group, and a relative increase of $12.5 \%$ in the control group. The intervention group utilized the CCR and the control group used did not use a formal screening tool. The CCR correctly identified all 23 clinically important cervical spine 
injuries with a sensitivity of $100 \%$. No fractures were missed and there were no adverse outcomes found. One limitation to the study was the baseline cervical spine imaging rates at the intervention and control sites were much lower than planned. Stiell et al. speculates that this was due to seven of the sites having participated in the previous validation study. Also, there is no way to confirm the patients did not go to a non-study hospital for imaging after discharge. The researchers also had difficulty with physician compliance with completing requisition forms and the rule was misinterpreted $17.1 \%$ of the time. According to Stiell et al. (2009), "widespread implementation of this rule could lead to reduced healthcare costs and more efficient patient flow in busy emergency departments worldwide" (p. 1).

A retrospective study from the UK was completed by Rethnam, Yesupalan, \& Gandham (2008) to determine if applying the CCR reduced the need for cervical spine radiography without missing significant cervical spine injuries. In this study, a convenience sample of 114 alert and stable patients at 2 hospitals who had cervical spine radiographs for suspected neck injuries were included. Based on chart review, patients were assessed and categorized as high or low risk using the CCR rule by two independent observers for each hospital. If the patient was low risk, records were checked for a neck range of motion assessment. Radiograph reports were also examined for positive or negative cervical spine injuries. It was found that of the 114 patients, 28 were high risk and required a radiological exam. There were 86 patients categorized as low risk and only 12 had neck range of motion assessments documented. The patients that did not have range of motion assessments were due to the over cautiousness of the attending clinician. According to this study, applying the CCR would have reduced the cervical 
spine radiography rates by $75.4 \%$ if all the patients in the low risk category had adequate range of motion on exam. Significant cervical spine injuries were found in two patients. Both patients were categorized in the high-risk category and were found when the CCR was applied. It was found that the CCR reduced the cervical spine radiography rates. This study was limited in that it was a retrospective design and the sample size was small including 114 patients. The $75 \%$ reduction rate could have been due to the fact that the researchers in the study presumed all patients in the low risk category had adequate neck range of motion. If neck range of motion was assessed, some of the patients may have required cervical spine radiographs, making the reduction rate smaller. Even with this missing data, the researchers noted the CCR still would have reduced the number of cervical spine radiographs (Rethnam, Yesupalan, \& Gandham, 2008).

Duane, et al. published a prospective evaluation of the CCR in 2011. The purpose of this study was to validate the CCR using CT as the gold standard to identify fractures. For this study, 3201 blunt trauma patients were screened using the CCR , the findings were compared with a complete cervical spine CT. Of these 3201 patients, 192 had a cervical spine fracture on CT vs 3009 without a fracture on CT. The sensitivity of the CCR was $100 \%$ with a specificity of $0.6 \%$. The positive predictive value was $6.03 \%$ and negative predictive value of $100 \%$. They concluded that the CCR is very sensitive but not very specific to determine the need for radiographic evaluation after blunt trauma. Duane et al. believe based on this study, the CCR should be utilized in emergency departments to improve specificity while maintaining sensitivity (Duane, et al., 2011, p. 352). 


\section{Clinician Adoption of the CCR}

In 2008, Eagles et al. set out to compare international awareness of the CCR by emergency physicians in Australia, Canada, the United Kingdom (UK) and United States (US). They also wanted to identify physician demographic and professional or practice setting that predict awareness and use of the rule. To do this, Eagles et al., conducted a self-administered email and postal survey between July 2005 and April 2008.

Emergency physicians were asked if they were aware of the CCR prior to receiving the survey, and how often they applied each rule when managing alert, stable patients with acute blunt trauma to the neck. If they were not currently applying the rule, they were asked if they would consider using it in the future and if not, why. The surveys included a copy of the CCR. The response rate was 54.8\%. A total of 1,297 survey questionnaires were included in the analysis. The percentage of physicians who were not aware of the CCR ranged from only $2 \%$ (in Canada) to $35 \%$ (in the US). The use of the rule was most prevalent in Canada with $73 \%$ use and lowest in the United States with only $30 \%$ of physicians using the CCR. The most commonly cited reason for not considering using the CCR in the future was current use of other guidelines or decision rules developed by another group. Full-time employees, younger age, and working in a teaching hospital were predictors of greater awareness (Eagles, et al., 2008). 


\section{Theoretical Framework}

The ACE Star Model of Knowledge Transformation was developed by Kathleen Stevens in 2004. The model was developed to bridge the gap between evidence-based nursing research and nursing practice. Knowledge transformation is defined as "the conversion of research findings from primary research results, through a series of stages and forms, to impact on health outcomes by way of evidence-based care" (Stevens, 2004). The ACE Star Model depicts a 5-point star, illustrating the five major stages of knowledge transformation. These five stages include discovery research, evidence summary, translation to guidelines, practice integration, and process/outcome evaluation (Stevens, 2004).

In the first star point, discovery research, new knowledge is obtained through primary research studies from descriptive studies to randomized control studies. This is considered a "knowledge generating stage" (Stevens, 2004).

The second star point, evidence summary, summarizes research knowledge into

single, meaningful statement. This summary includes evidence synthesis, meta-analysis, integrative review, review of literature, state of the science review, and systematic reviews of randomized control trials. The evidence summaries produce new knowledge by combining previous studies and identifying reproducibility of results, which is why this is also considered a knowledge generating stage (Stevens, 2004).

The third star point, translation to guidelines, transforms the evidence summaries from above into practice recommendations and integration into practice. Practice 
recommendations are also considered clinical practice guidelines. Clinical practice guidelines aid health care workers to make informed, evidence based decisions.

The fourth star point, practice integration, involves the implementation of the latest evidence based practice guidelines from above. This also involves the individual factors that affect individual health care providers, patients, and organizations that may affect the integration of change to evidence based practice (Stevens, 2004).

The fifth and final star point is process, outcome evaluation. This stage includes the evaluation of the impact on patient outcomes, provider and patient satisfaction, efficacy, efficiency, cost, and health status. The ideal outcome is evidence-based quality improvement of healthcare (Stevens, 2004).

The ACE Star Model of Knowledge Transformation can be applied to the use and application of the Canadian Cervical Spine Rule (CCR) in this retrospective chart review. The CCR has surpassed the second star point, evidence summary. As noted in the literature review above, the CCR has a significant amount of research and systematic reviews to support its use and application. The CCR has also surpassed the third star pint, translation to guidelines. The guidelines have been created but have not been fully integrated into practice in emergency department nationally. With further evaluation of the benefits and application of the CCR, this tool can proceed to the fourth star point, practice integration. The ACE Star Model of Knowledge Transformation fits well with the clinical utilization of the CCR with suspected cervical spine injuries, as well as supporting this retrospective chart review. 


\section{Method}

\section{Purpose}

The purpose of this project was to complete a retrospective chart review to evaluate the number of cervical spine radiographs that could have been prevented by applying the Canadian Cervical Spine Rule in adults admitted to the emergency department with a suspected cervical spine injury.

\section{Design}

The project design was a retrospective chart review, which took place at a level 2 trauma center emergency department in a community teaching hospital reviewing patient records and radiological findings in relation to the potential benefit of using the CCR rule in CSI assessment.

\section{Sample and site}

The sample was a non-probability convenience sample of patients whom have had a cervical spine radiograph in the emergency department. Inclusion criteria included patients age 18 and older. Patients must have had a cervical spine radiograph to rule out CSI during their stay in the emergency department. Exclusion criteria includes patients under the age of 18, non-trauma cases, Glasgow coma score $<15$, unstable vital signs, acute paralysis, known vertebral disease, or previous surgery of the cervical spine. One hundred charts were reviewed and 60 patient records were included in the study. The site was a level 2 trauma center emergency department an academic medical center located in the New England area. A retrospective chart review was performed from January $1^{\text {st }}$, 2016 to June $30^{\text {th }}, 2016$. 


\section{Procedures}

The proposed study was submitted for approval to the Rhode Island College and Lifespan Investigational Review Boards. Approval was obtained from The Miriam Hospital Emergency Department, the Director of Nursing, as well as the Quality Assurance and Safety Coordinator in the Radiology Department at Hospital. Patients who have had a cervical spine radiograph between January $1^{\text {st }}, 2016$ and June $30^{\text {th }}, 2016$ were identified via radiology records. These records were obtained from the quality assurance and safety coordinator in the radiology department. A list of medical record numbers of patients who had a cervical spine CT were provided from the Quality Assurance and Safety Coordinator in the radiology department. After reviewing the record, the medical record number was removed, and only de-identified information was collected on the data collection sheet. The researcher then reviewed the chart for patient age, gender, mechanism of injury, paresthesia in extremities, sitting position in emergency department, walking at any time, delayed onset of neck pain, presence of midline cervical spine tenderness, active range of motion, ED length of stay, and radiographic result for presence or absence of cervical spine injury, as these elements are necessary to the utilization of the CCR. All data was entered in the data collection sheet (Appendix C). A potential barrier to data collection includes inadequate provider physical exam documentation. The author is a Lifespan employee and is familiar with the electronic medical record system utilized at the Emergency Department setting, this further supported the chart review. 


\section{Measurement}

Data collected from the medical records was collected according to the CCR to determine if the cervical collar could have safely been removed without the use of a CT. The author identified the number of CCR elements that were documented in the physical exam. The author quantified the number of charts that met high risk criteria, number of charts that met low risk criteria, number of charts with documented assessment with neck range of motion, and number of radiologist confirmed normal and abnormal CT scans. The researcher then reviewed the chart for patient age, gender, mechanism of injury, paresthesia in extremities, sitting position in emergency department, walking at any time, delayed onset of neck pain, presence of midline cervical spine tenderness, active range of motion, length of ED stay, and radiographic reports for presence or absence of cervical spine injury, as these elements are necessary to the utilization of the CCR.

\section{Data Analysis}

Descriptive statistics will be used, describing the percent, mean and standard deviation of the statistics calculated.

\section{Ethical Considerations}

Identifying patient information was not collected and patient unique identifiers remained anonymous. Data was collected using a secure internet source. Data collection tool was stored in a locked, password protected electronic device. 


\section{Results}

A total of 100 medical records were reviewed to obtain a cohort of 60 subjects who underwent a cervical spine radiograph in the Emergency Department to rule out a cervical spine injury. Forty records were excluded from the study. One for age less than 18 years, three excluded as they were inpatient and not in the Emergency Department, 18 were excluded as non trauma cases, one excluded as they had a previous cervical spine surgery, 11 excluded for known vertebral disease, and 6 excluded for a Glascow Coma Scale of less than 15.

Of the 60 medical records reviewed, 30 met high risk criteria. High risk criteria according to the CCR includes, age greater than or equal to 65 years, paresthesias in extremities, or dangerous mechanism of injury (fall from elevation greater than or equal to 3 feet or 5 stairs, axial load to head, motor vehicle collision high speed over $100 \mathrm{~km} / \mathrm{h}$, rollover, ejection, motorized recreational vehicles, bicycle struck or collision). Twentythree records were classified as high risk for age greater than or equal to 65 years, 6 were high risk for evidence of paresthesias, and one was classified as high risk for dangerous mechanism of injury.

Of the 60 records reviewed, 30 did not meet high risk criteria. All 30 records had at least one low risk factor that allows safe assessment of range of motion. A low risk factor to allow safe assessment of range of motion included simple rear ended collision (excludes pushed into oncoming traffic, hit by bus or large truck, rollover, hit by high speed vehicle), sitting position in the emergency department, walking at any time, delayed onset of neck pain, and absence of midline cervical spine tenderness. Seventeen 
subjects were in a simple rear ended collision, 20 were walking at any time, nine had delayed onset of neck pain, and two had an absence of midline cervical spine tenderness.

Of the 30 records, which did not meet high risk criteria and had at least one low risk factor allowing safe assessment of range or motion, one was unable to actively rotate their neck. Eight records had documented normal range of motion in the musculoskeletal assessment, one had documented normal neck range of motion, and 19 did not comment on musculoskeletal or neck range of motion.

Of the 60 records reviewed, there was no radiographic evidence of a clinically important cervical spine injury including fracture, dislocation, or ligamentous instability detectable by diagnostic imaging and requiring surgical or specialist follow up. 


\section{Summary and Conclusions}

The purpose of this project was to complete a retrospective chart review to evaluate the number of cervical spine radiographs that could have been prevented by applying the Canadian Cervical Spine Rule in adults admitted to the emergency department with a suspected cervical spine injury. Cervical spine immobilization with the use of rigid cervical collars can be uncomfortable, delays hospital transport, and has a modest morbidity. Data collection included age, gender, high risk factors or low risk factors, as described by the CCR, ability to actively rotate neck, and radiologist confirmed CT interpretation. The use of the CCR has been shown to decrease the patient emergency department length of stay, provide more comfort for the patient, and decrease the health care costs of unnecessary imaging.

Of the 60 records included in the study, half met high risk criteria requiring cervical spine radiography. Of the 30 records meeting low risk criteria, only 2 charts had complete documentation including neck range of motion. One had normal range of motion and one was unable to actively rotate neck. There were 8 records that documented "normal range of motion" but did not specifically note normal neck range of motion. It is difficult to definitively state that $28 \mathrm{CTs}$ could have been prevented, as there was inadequate neck range of motion documentation. This finding is similar to that in the 2008 study by Rethnam, Yesupalan, and Gandham. In this study they inferred that all patients that did not have neck range of motion documented would have had normal neck range of motion. If this is the case in the current study, applying the CCR would have reduced the cervical spine radiography rates by $48 \%(29 / 60)$. 
There were no cervical spine injuries detected by CT in this chart review. As with previous studies, the data shows that the CCR maintains is sensitivity but has low specificity. It remains that ED clinicians still rely on radiology evidence to rule out cervical spine injury rather than evidence based clinical assessment recommended by specialty societies.

There are several limitations to this study. The greatest is the lack of complete physical exam documentation to accurately apply the CCR. Second, this is a small sample size of only 60 medical records.

In conclusion, based on this small sample, patients presenting to the ED with a possible cervical spine injury would experience a significant reduction in cervical spine radiographs if the patients were accurately screened using the CCR. 


\section{Recommendations and Implications for Advanced Nursing Practice}

Every day patients present to the emergency department with suspected neck injury. With the increasing cost of healthcare, and significant ED overcrowding, it is vital for providers to base care on evidence based guidelines and recommendations. The evidence based, specialty society recommended Canadian Cervical Spine rule has proven sensitivity to clear the cervical spine without the use of imaging. The use of this simple clinical assessment tool can aid in decreasing ED length of stay, minimize healthcare costs, and save the patient from unnecessary radiation exposure.

As evidenced in this study, there could have been a $48 \%$ reduction in cervical spine radiographs if the CCR was applied and neck range of motion was documented. Despite the increase in litigation in healthcare, it is important for providers not to solely depend on radiographic evidence to rule out cervical spine injury. The CCR has been tested multiple times, specialty society recommended and has consistently been proven to maintain its sensitivity. Despite its lower specificity, it continues to decrease the number of cervical spine radiographs ordered.

Further research is needed to truly assess the number of cervical spine radiographs that could have been prevented. It cannot be generalized due to incomplete documentation regarding neck range of motion in this small sample. There have been very few studies to examine if emergency department nurses can safely apply the CCR. Further research can assess if ED nurses can safely apply the CCR. To further improve patient comfort and decrease length of stay. 
Advanced practice nurses are in a position to educate other ED providers on the accurate use of this evidenced based, clinical assessment tool. APRNs can bring this research and evidence to the ED multidisciplinary team to enhance assessment, documentation and screening of patients presenting with possible cervical spine injuries. Further consideration of this highly sensitive clinical decision making tool is warranted in this country. 


\section{References}

California Health Care Foundation. (2016). Health care costs 101: ACA spurs modest growth. Retrieved from http://www.chcf.org/publications/2016/05/health-carecosts-101

Benayoun, M., Allen, J., Lovasik, B., Uriell, M., Spandorfer, R., and Holder, C. (2016). Utility of computed tomographic imaging of the cervical spine in trauma evaluation of ground-level fall. Journal of Trauma Acute Care Surgery, 81(2). 339-344. doi: 10.1097/TA.0000000000001073

Cuong Pham, J., Patel, R., Millin, M., Kirsch, T., and Chanmugam, A. (2006). The effects of ambulance diversion: A comprehensive review. Academic Emergency Medicine, 13(11). 1220-1227. doi 10.1197/j.aem.2006.05.024

Duane, T., Wilson, S., Mayglothling, J., Wolfe, L., Aboutanos, M., Whelan, J...Ivatury, R. (2011). Canadian cervical spine rule compared with computed tomography: A prospective analysis. Journal of Trauma, Injury, Infection and Critical Care, 71(2). 352-357. doi 10.1097/TA.0b013e318220a98c

Eagles, D., Stiell, I, Clement, C., Brehaut, J., Taljaard, M., Kelly, A... Perry, J. (2008). International survey of emergency physicians' awareness and use of the Canadian cervical spine rule and the Canadian computed tomography head rule. Society for Academic Emergency Medicine, 15(12). 1256-1261. doi: 10.111/j.15532712.2008.00265.x

Hadley, M., and Walters, B. (2001) Guidelines for the management of acute cervical spine and spinal cord injuries. American Association of Neurologic Surgeons Institute of Medicine, of the National Academies. (2006). The future of emergency care: Key findings and recommendations. Retrieved from 
http://nationalacademies.org/hmd/ /media/Files/Report\%20Files/2006/HospitalBased-Emergency-Care-At-the-BreakingPoint/EmergencyCareFindingsandRecs.pdf

Massachusetts office of Emergency Medical Services. (2015). Emergency medical services pre-hospital statewide treatment protocols. Bureau of Healthcare Safety and Quality. Boston, Massachusetts.

Michaleff, Z., Maher, C., Verhagen, A., Rebbeck, T., Lin, C. (2012). Accuracy of the Canadian C-spine rule and NEXUS to screen for clinically important cervical spine injury in patients following blunt trauma: a systematic review. Canadian Medical Association Journal, 184(16). E867-E876.

Rethnam, U., Yesupalan, R., Gandham, G. (2008). Does applying the Canadian Cervical Spine rule reduce cervical spine radiography rates in alert patients with blunt trauma to the neck? A retrospective analysis. BMC Medical Imaging, 8(12). http://www.biomedcentral.com/1471-2342/8/12

Schuur, J., Carney, D., Everett, L., Raja, A., Michael, J., Ross, N., \& Venkatesh, A. (2014). A top-five list for emergency medicine: A pilot project to improve the value of emergency care. Journal of the American Medical Association, 174(4), 509-515. doi: 10.1001

Spinal Cord Injury Model System. (2015). Understanding spinal cord injury: Part 1- The body before and after injury. Retrieved from http://www.msktc.org/lib/docs/Factsheets/SCI_Understand_Spin_Crd_Inj_Prt1.pd $\mathrm{f}$ 
Squires, D., \& Anderson, C. (2015). The commonwealth fund. U.S. Health Care from a Global Perspective: Spending, Use of Services, Prices, and Health in 13 Countries. Retrieved from http://www.commonwealthfund.org/publications/issue-briefs/2015/oct/us-healthcare-from-a-global-perspective

Stevens, K. R. (2004). ACE Star Model of EBP: Knowledge Transformation. Academic Center for Evidence-based Practice. The University of Texas Health Science Center at San Antonio. Retrieved from http://nursing.uthscsa.edu/onrs/starmodel/star-model.asp

Stiell, I., Clement, C., Grimshaw, J., Brison, R., Rowe, B., Schull, M., ... Wells, G. (2009). Implementation of the Canadian C-Spine Rule: prospective 12 centre cluster randomized trial. BMJ, doi: http://dx.doi.org/10.1136/bmj.b4146. 


\section{Appendix A}

The Canadian C-Spine rule utilized to decrease the number of unnecessary radiographs and decrease patient immobilization times (Stiell et al., 2009).

\section{Canadian C-Spine Rule}

For alert (Glasgow coma score $=15$ ) and stable trauma patients where cervical spine injury is a concern

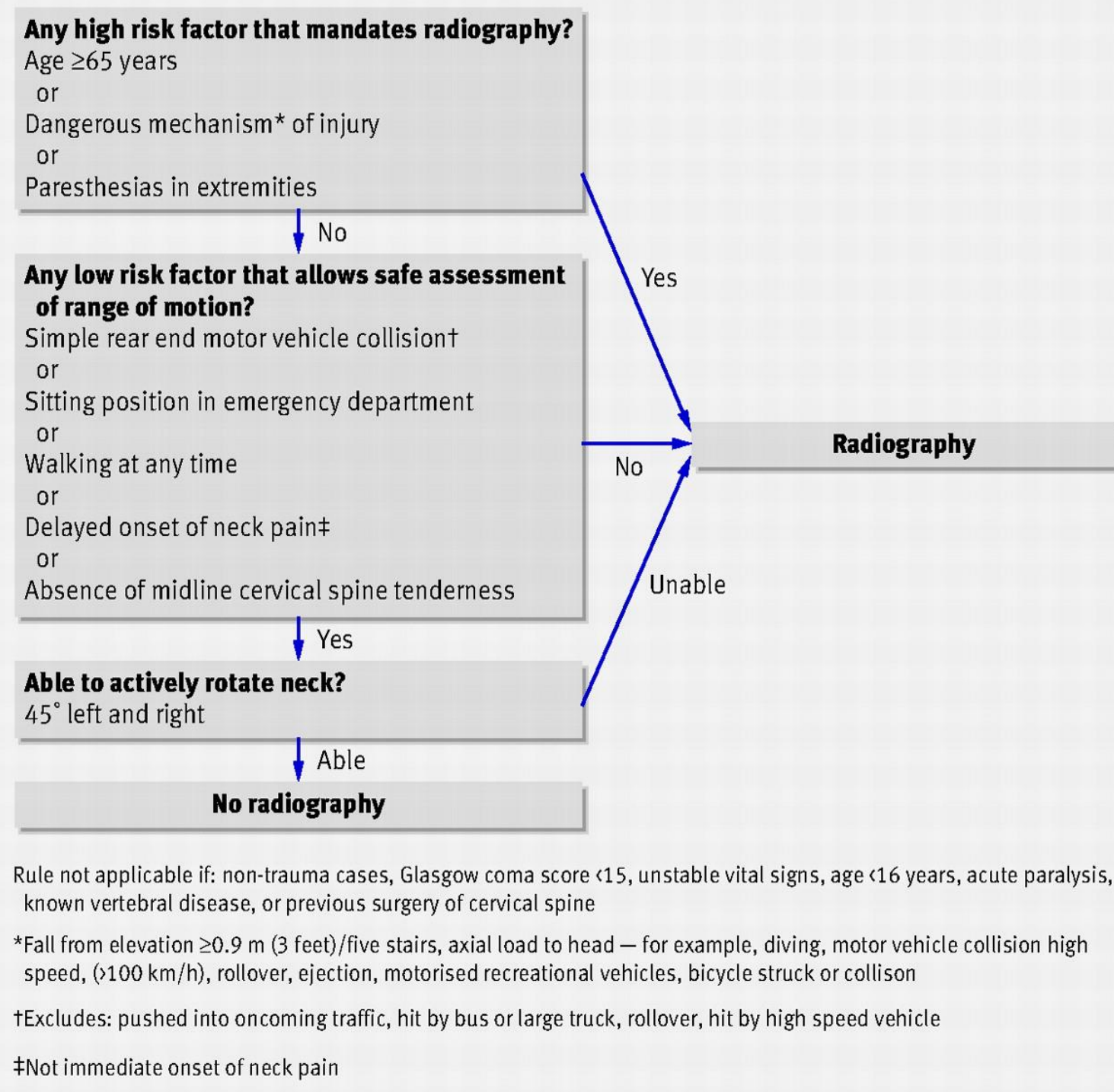




\section{Appendix B}

NEXUS low-risk criteria utilized to decrease the number of unnecessary radiographs and decrease patient immobilization times (Michaleff, Maher, Verhagen, Rebbeck, Lin, (2012).

\section{Box 1: National Emergency X-Radiography Utilization Study (NEXUS) low-risk criteria}

Cervical Spine radiography is indicated for patients with neck trauma unless they meet ALL of the following criteria:

- No posterior midline cervical-spine tenderness

- No evidence of intoxication

- A normal level of alertness (score of 15 on the Glasgow Coma Scale)

- No focal neurologic deficit

- No painful distracting injuries 


\section{Appendix C}

\section{Data collection tool}

\begin{tabular}{|c|c|c|c|c|c|c|c|}
\hline & Age & Gender & High risk factor* & Low risk factors* & Midline tenderness & Able to actively | Radiology result & ED LOS (min) \\
\hline 1 & & & & & & & \\
\hline 2 & & & & & & & \\
\hline 3 & & & & & & & \\
\hline 4 & & & & & & & \\
\hline 5 & & & & & & & \\
\hline 6 & & & & & & & \\
\hline 7 & & & & & & & \\
\hline 8 & & & & & & & \\
\hline 9 & & & & & & & \\
\hline 10 & & & & & & & \\
\hline 11 & & & & & & & \\
\hline 12 & & & & & & & \\
\hline 13 & & & & & & & \\
\hline 14 & & & & & & & \\
\hline 15 & & & & & & & \\
\hline 16 & & & & & & & \\
\hline 17 & & & & & & & \\
\hline 18 & & & & & & & \\
\hline 19 & & & & & & & \\
\hline 20 & & & & & & & \\
\hline 21[ & & & & & & & \\
\hline 22 & & & & & & & \\
\hline 23 & & & & & & & \\
\hline 24 & & & & & & & \\
\hline 25 & & & & & & & \\
\hline 26 & & & & & & & \\
\hline 27 & & & & & & & \\
\hline 28 & & & & & & & \\
\hline 29 & & & & & & & \\
\hline
\end{tabular}

